05.6;09.1

\title{
Излучение Вавилова-Черенкова и импульсная катодолюминесценция в полиметилметакрилате при возбуждении субнаносекундным пучком электронов
}

\author{
(C) В.Ф. Тарасенко ${ }^{1,2}$, E.Х. Бакшт ${ }^{1}$, М.В. Ерофреев ${ }^{1}$ \\ ${ }^{1}$ Институт сильноточной электроники СО РАН, Томск, Россия \\ ${ }^{2}$ Национальный исследовательский Томский государственный университет, Томск, Россия \\ E-mail:VFT@loi.hcei.tsc.ru
}

Поступило в Редакцию 28 октября 2020г.

В окончательной редакции 13 ноября 2020 г.

Принято к публикации 27 ноября 2020 r.

Исследованы спектральные и амплитудно-временны́е характеристики излучения полиметилметакрилата при возбуждении пучками электронов с энергией электронов до 300 и $450 \mathrm{keV}$. C субнаносекундным временны́ разрешением зарегистрированы импульсы излучения Вавилова-Черенкова и катодолюминесценции. Показано, что интенсивность излучения импульсной катодолюминесценции в видимой и ближней ультрафиолетовой областях спектра при энергии электронов сотни $\mathrm{keV}$ существенно превышает интенсивность излучения Вавилова-Черенкова.

Ключевые слова: пучок электронов, излучение Вавилова-Черенкова, импульсная катодолюминесценция, фотовозбуждение.

DOI: 10.21883/PJTF.2021.06.50749.18601

Полиметилметакрилат (ПММА, оргстекло) широко используется для регистрации высокоэнергетических частиц, в том числе в медицинской дозиметрии [1-5]. Это обусловлено доступностью данного материала и сравнительно высокой интенсивностью излучения ПММА при воздействии электронов и других частиц [1-7]. Считается [1-4], что при высоких энергиях электронов излучение ПММА в основном состоит из излучения Вавилова-Черенкова (ИВЧ). Однако приводимые спектры излучения ПММА существенно отличаются от спектров ИВЧ (см., например, [4,6,7]). Из теории [8] хорошо известно, что интенсивность ИВЧ в области прозрачности образцов возрастает с уменьшением длины волны излучения. Расчеты спектров ИВЧ для различных веществ при энергиях электронов в десятки-сотни $\mathrm{keV}$ приведены в [6,9-11].

Экспериментально (при возбуждении пучком электронов с энергией, составляющей сотни $\mathrm{keV}$ и $6 \mathrm{MeV}$ ) спектры ИВЧ для образцов из алмаза, кварца и сапфира были получены в работах [9-11], где демонстрировались основные отличительные свойства ИВЧ. К ним относятся наличие для появления ИВЧ пороговой энергии у заряженных частиц, увеличение интенсивности ИВЧ с уменьшением длины волны, безынерционность эффекта Вавилова-Черенкова относительно импульса возбуждения и наличие угла между направлением ИВЧ и траекторией движения заряженных частиц, который зависит от энергии частиц.

Спектр излучения, приведенный в [4], имел полуширину основной полосы $\sim 225 \mathrm{~nm}$ с максимумом на длине волны $\sim 510 \mathrm{~nm}$. Также была зарегистрирована вторая полоса меньшей интенсивности с максимумом на длине волны $\sim 650 \mathrm{~nm}$. Эти данные, в том числе согласно мнению авторов [4], отличались от спектров ИВЧ. В [6,7] было показано, что при возбуждении ПММА пучком электронов с энергией до $300 \mathrm{keV}$ на спектрограммах видны полосы только импульсной катодолюминесценции (ИКЛ), которые имели максимум для основной полосы на длине волны $\sim 490 \mathrm{~nm}$ при ее полуширине $\sim 100 \mathrm{~nm}$. Также отметим, что интенсивность ИКЛ в условиях [6,7] зависела от производителя ПММА и была минимальной в образцах высокой чистоты.

Широкий набор спектров излучения ПММА для различных энергий электронов и рентгеновских квантов приведен в [5], в том числе для пиковых напряжений на рентгеновской трубке 75, 125 и $250 \mathrm{kV}$. В представленных спектрах ИВЧ в области 400-700 nm интенсивность излучения возрастала с уменьшением длины волны до $400 \mathrm{~nm}$, что соответствует поведению черенковского излучения. Данные для длин волн короче $400 \mathrm{~nm}$ в [5] отсутствуют. Однако подобную зависимость имели и спектры ИКЛ, приводимые в этой работе. Как уже отмечалось выше, в [6,7] было показано, что при возбуждении ПММА пучком электронов с энергией до $300 \mathrm{keV}$ наблюдалась полоса ИКЛ, интенсивность которой уменьшается начиная с длины волны $490 \mathrm{~nm}$.

Цель настоящей работы - провести исследования спектральных и амплитудно-временны́х характеристик излучения ПММА при возбуждении пучком электронов субнаносекундной длительности с различной энергией электронов в диапазоне сотен $\mathrm{keV}$, а также получить данные о соотношении интенсивностей излучения Вавилова-Черенкова и импульсной катодолюминесценции. 


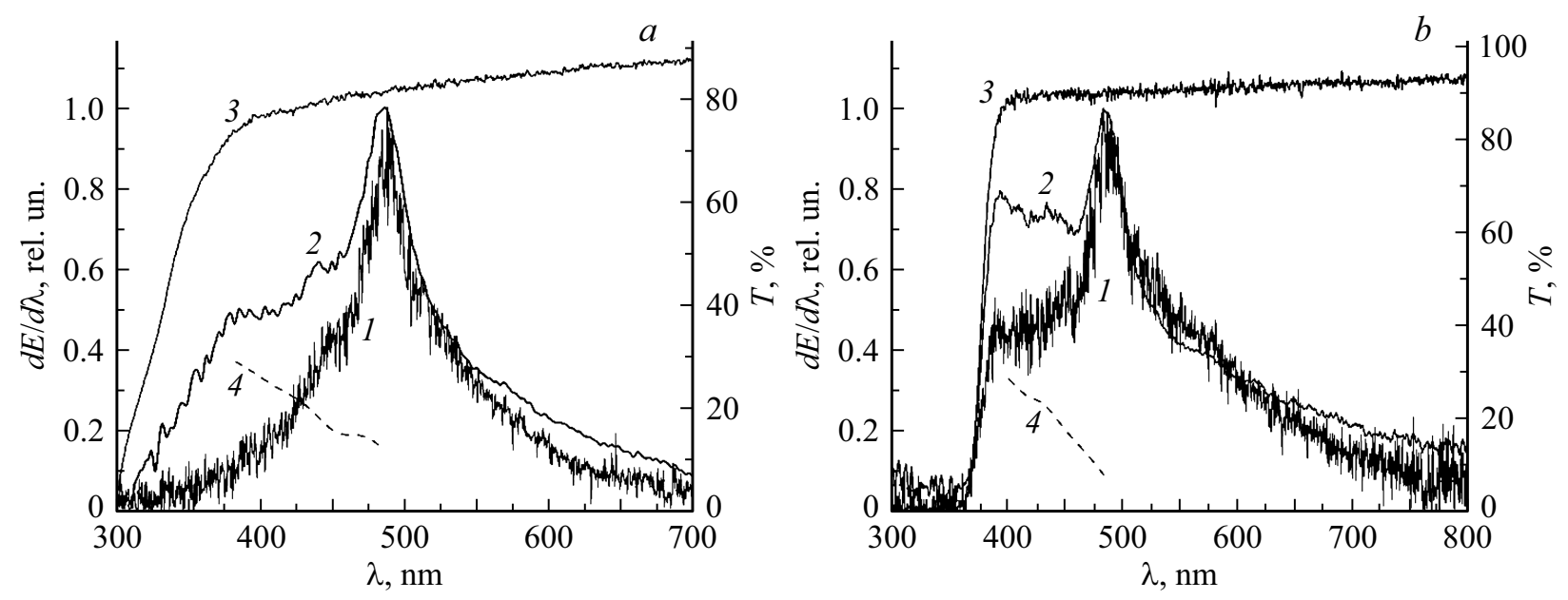

Рис. 1. Спектры излучения ПММА, характерные для образцов первой $(a)$ и второй $(b)$ групп, при возбуждении пучком электронов ускорителей с генератором СЛЭП-150 (1) и с генератором ГИН-1000 (2). Кривые 3 соответствуют коэффициенту пропускания данных образцов, а кривые 4 показывают добавку в общий спектр излучения Вавилова-Черенкова.

Для воздействия на ПММА использовались два ускорителя с генераторами СЛЭП-150 и ГИН-1000, которые обеспечивали длительность импульса тока пучка $\sim 350$ ps. Первый (СЛЭП-150) формировал пучок электронов с энергией до $300 \mathrm{keV}$ при плотности тока $\sim 200 \mathrm{~A} / \mathrm{cm}^{2}$. Генератор ГИН-1000 второго ускорителя состоял из трех секций и формировал пучок электронов с энергией до $450 \mathrm{keV}$ при плотности тока $\sim 2 \mathrm{kA} / \mathrm{cm}^{2}$. Ток пучка электронов измерялся с помощью конического коллектора с диаметром приемной части $15 \mathrm{~mm}$, который имел временно́е разрешение не хуже 100 ps. При измерениях тока пучка пространство между фольгой и коллектором откачивалось форвакуумным насосом. Для ослабления тока электронного пучка при измерениях коллектором применялись металлические сетки с известной прозрачностью. Образцы ПММА устанавливались за AlMg-фольгой толщиной $40 \mu \mathrm{m}$ перпендикулярно оси ускорителя. Всего было исследовано восемь образцов толщиной $0.9,3,4,6$ и $10 \mathrm{~mm}$ от различных производителей. Образцы высокой чистоты были изготовлены в Сибирском физико-техническом институте (Томск).

Спектры пропускания и излучения ПММА регистрировались с помощью спектрометра Ocean Optics HR2000+ES (спектральный диапазон измерений 200-1000 nm, разрешение $0.9 \mathrm{~nm}$ ), излучение на который подавалось по световоду P600-1-SR (Ocean Optics, Inc.). Амплитудно-временны́е характеристики излучения ПММА регистрировались фотодиодом PD025 (катод LNS20 компании Photek, время нарастания переходной характеристики $\sim 80 \mathrm{ps}$ ), который помещался в алюминиевый стакан. Сигналы с фотодиода, емкостных делителей генераторов и коллектора подавались на цифровой осциллограф Keysight DSO-X6004A (6 GHz и 20 S/ns) через высокочастотные кабели 5D-FB PEEG (Radiolab) длиной $1.2 \mathrm{~m}$. Для ослабления сигналов использовались ослабители 142-NM (Barth Electronics, $30 \mathrm{GHz}$ ), позволяющие регистрировать без существенных искажений импульсы напряжения с фронтом до 20 ps.

Спектральные измерения показали, что образцы по их спектральным характеристикам можно разбить на две группы. Образцы первой группы имели пропускание начиная с $300 \mathrm{~nm}$, а второй — с $370 \mathrm{~nm}$. Кроме того, при энергии электронов до $300 \mathrm{keV}$ в первой группе образцов, как и в работах [6,7], вторая полоса с коротковолновым максимумом $\sim 400 \mathrm{~nm}$ отсутствовала (рис. 1, $a$, кривая 1).

Наблюдаемая полоса на рис. 1, $a$ (кривая 1), как уже было показано нами в [6,7], относится к ИКЛ. Доминирование ИКЛ в этих условиях обусловлено малым коэффициентом преломления света в ПММА (1.49), соответственно высокой пороговой энергией электронов для появления ИВЧ $(178 \mathrm{keV})$ и малым числом электронов в пучке с энергией, которая превышает пороговую (не более 20\% с генератором СЛЭП-150).

При увеличении плотности тока пучка на порядок и энергии электронов (число электронов с энергией, превышающей пороговую для ИВЧ, было около 50\%) с генератором ГИН-1000 спектр излучения изменялся (кривая 2), и в области спектра с длинами волн меньше $450 \mathrm{~nm}$ появлялась вторая полоса меньшей интенсивности. Коротковолновая граница этой полосы определялась поглощением в ПММА (кривая 3), которое начиная с $300 \mathrm{~nm}$ обрезало все излучение. Нормируя максимумы спектров излучения 1 и 2 на единицу и определяя разность между ними, можно восстановить форму спектра появившейся полосы (см. кривую 4). Данная полоса, которая возникла при увеличении энергии электронов, по нашему мнению состоит в основном из ИВЧ. Спектральная плотность энергии излучения этой полосы $d E / d \lambda$ в области прозрачности образца 

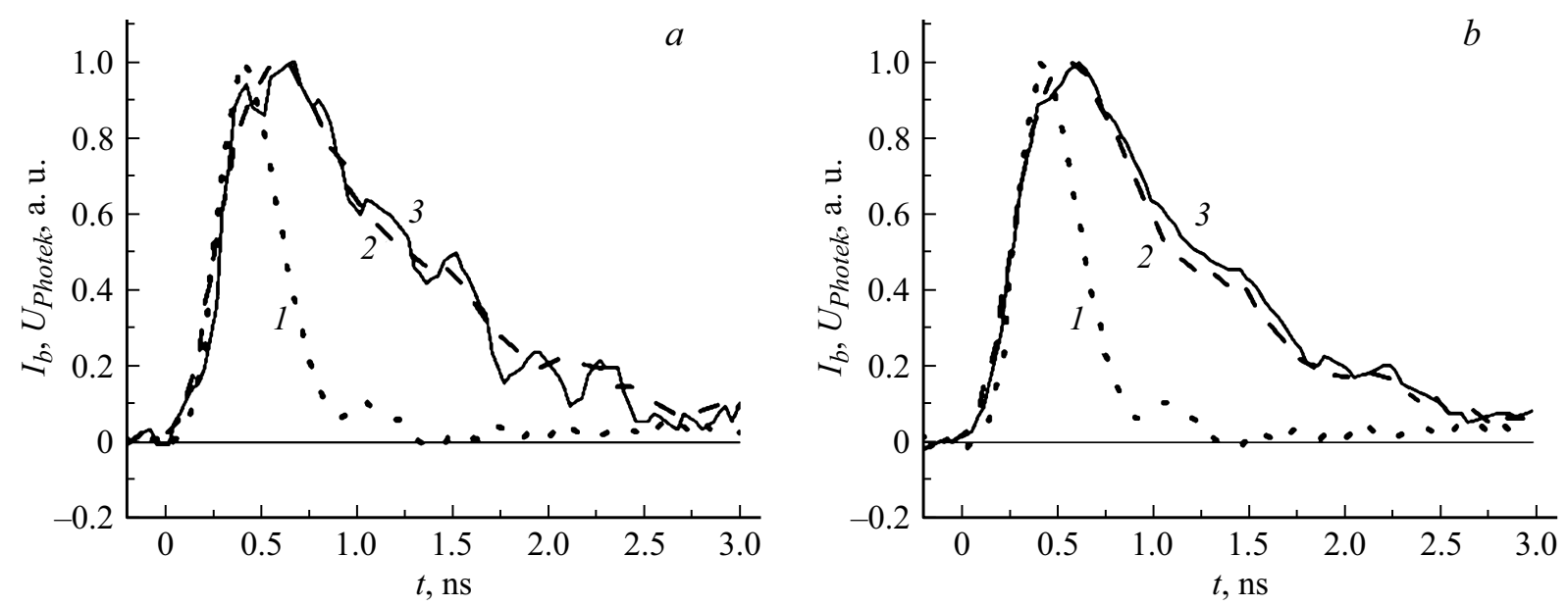

Рис. 2. Осциллограммы импульсов тока пучка $(1)$ и излучения $(2,3)$ ПММА, характерные для образцов первой $(a)$ и второй $(b)$ групп (2 - при возбуждении пучком электронов от генератора ГИН-1000 без фильтра, 3 - с фильтром УФС-1).

возрастает с уменьшением длины волны, что является одним из признаков, которые характеризуют ИВЧ.

Характерные спектры для второй группы образцов показаны на рис. $1, b$. Эти образцы имели сильное поглощение начиная с длины волны $370 \mathrm{~nm}$, и при возбуждении генератором СЛЭП-150 в них наблюдалось две полосы излучения. Обе полосы регистрировались при сравнительно низких энергиях электронов, в том числе, как показали дополнительные измерения, меньших пороговых для ИВЧ в ПММА. Коротковолновая граница второй полосы совпадала с границей пропускания в образцах второй группы. Обе эти полосы (кривая 1 на рис. $1, b$ ) обусловлены ИКЛ. Данный вывод подтверждает работа [6], в которой вторая полоса регистрировалась при облучении этих образцов излучением эксилампы с длиной волны $222 \mathrm{~nm}$ (см. рис. 12, $b$ в [6]). Кроме того, эта полоса с максимумом на длине волны $400 \mathrm{~nm}$ отсутствовала в образцах первой группы при возбуждении пучком электронов от генератора СЛЭП-150. Вторую полосу нельзя относить к ИВЧ, так как все исследованные образцы были изготовлены из ПММА с одинаковым коэффициентом преломления.

При увеличении энергии электронов в пучке (рис. $1, b$, кривая 2) в спектре излучения появилось ИВЧ. Дополнительную полосу в УФ-области мы также выделили, нормируя максимумы интенсивности излучения, полученные с двумя ускорителями, на единицу и определяя разность (см. кривую 4 на рис. $1, b$ ). Интенсивность излучения выделенной полосы увеличивается при уменьшении длины волны, что характерно для ИВЧ, но зависимости близки к линейным. Как известно [8], теория дает увеличение интенсивности ИВЧ обратно пропорционально кубу длины волны. Однако этот вывод справедлив для „тонких“ образцов, при прохождении которых направление движения и энергия электронов существенно не меняются. В данных экспериментах использовались „толстые“ (для энергии электронов в сотни $\mathrm{keV}$ ) образцы. Соответственно энергия электро- нов при торможении в образцах изменялась, что приводило к изменению угла между траекторией электронов и конусом ИВЧ. Кроме того, направление движения электронов изменялось при их торможении в образцах. Все это повлияло на наклон зависимости интенсивности ИВЧ от длины волны.

Для дополнительной проверки наличия в этих условиях ИВЧ были измерены амплитудно-временнь́е характеристики свечения образцов обеих групп с высоким временны́м разрешением. Спектры излучения регистрировались в широкой спектральной области 300-550 nm и в сравнительно узкой спектральной области, которая вырезалась с помощью фильтра УФС-1, имеющего пропускание в области 300-400 nm. Соответственно для образцов первой группы, имеющих коротковолновую границу поглощения $300 \mathrm{~nm}$ (рис. 1, $a$ ), регистрировалось излучение в области $300-400 \mathrm{~nm}$, а для образцов второй группы, имеющих коротковолновую границу поглощения $370 \mathrm{~nm}$ (рис. 1, b), - в области 370-400 nm. Как следует из рис. 1, при использовании фильтра доля регистрируемого ИВЧ относительно ИКЛ должна увеличиваться. Полученные результаты приведены на рис. 2.

Видно, что длительность импульса излучения составляет $\sim 1 \mathrm{~ns}$ при регистрации излучения как в широкой области спектра, так и в сравнительно узкой области для обеих групп образцов. Длительность импульса тока пучка на полувысоте при этом равнялась $370 \mathrm{ps}$. Таким образом, основная доля регистрируемого излучения принадлежит ИКЛ даже при максимальной энергии электронов $450 \mathrm{keV}$, когда примерно у половины электронов пучка энергия превышала пороговую для ИВЧ и плотность тока пучка составляла $\sim 2 \mathrm{kA} / \mathrm{cm}^{2}$. Вместе с тем на импульсах излучения для образцов второй группы виден первый пик, соответствующий максимуму тока пучка, а для образцов первой группы - характерный изгиб, соответствующий максимуму тока пучка. Из этого следует, что в формировании импульса излучения 
участвуют два процесса (ИВЧ и ИКЛ), которые имеют разные длительности. Отсутствие явно выраженного пика для второй группы образцов связано с поглощением образцов в области спектра короче $370 \mathrm{~nm}$ и соответствующим уменьшением доли регистрируемого сигнала ИВЧ. Также наличие ИВЧ косвенно подтверждает совпадение фронта импульса тока пучка и излучения. Отметим, что при регистрации импульсов излучения фотодиодом, который помещался в защитный экран, использовался аттенюатор Barth Electronics 142-NM с полосой пропускания до $30 \mathrm{GHz}$, ослабляющий сигналы в 20 раз, что позволило подавить влияние электрических помех. В экспериментах также было установлено, что оба типа излучения имеют малое время высвечивания и их интенсивность растет с увеличением плотности тока пучка.

Таким образом, проведенные исследования показали, что при энергиях электронов в несколько сотен $\mathrm{keV}$ в спектрах излучения на фоне ИКЛ можно регистрировать полосу ИВЧ, но она уступает по интенсивности ИКЛ. Следует отметить важное свойство образцов из ПММА, а также из других материалов, в которых имеются полосы фотолюминесценции в УФ- и видимой областях спектра, а энергии электронов пучка достаточно для возникновения ИВЧ. Благодаря увеличению мощности излучения с уменьшением длины волны, ИВЧ даже в области поглощения ПММА может обеспечить его фотовозбуждение, что усиливает интенсивность полос, наблюдаемых при ИКЛ. Данный эффект был обнаружен

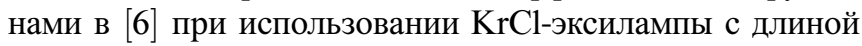
волны $222 \mathrm{~nm}$ и подтвержден в работах $[12,13]$ при использовании коротковолнового излучения с другими длинами волн, а также с другими материалами образцов. В настоящей работе при возбуждении ПММА излучением эксиламп с длиной волны 222 и $283 \mathrm{~nm}$ также регистрировались полосы фотолюминесценции в области полос ИКЛ. Соответственно сравнительно высокая интенсивность ИКЛ, которая следует из экспериментов с фотодиодом, при увеличении энергии электронов и плотности тока пучка отчасти связана с возбуждением фотолюминесценции за счет ИВЧ в области поглощения ПММА.

\section{Финансирование работы}

Работа выполнена в рамках проекта Российского научного фонда № 18-19-00184.

\section{Конфликт интересов}

Авторы заявляют, что у них нет конфликта интересов.

\section{Список литературы}

[1] A.M. Frelin, J.M. Fontbonne, G. Ban, J. Colin, M. Labalme, A. Batalla, A. Isambert, A. Vela, T. Leroux, Med. Phys., 32 (9), 3000 (2005). https://doi.org/10.1118/1.2008487
[2] K.W. Jang, T. Yagi, C.H. Pyeon, W.J. Yoo, S.H. Shin, C. Jeong, B.J. Min, D. Shin, T. Misawa, B. Lee, J. Biomed. Opt., 18 (2), 027001 (2013). https://doi.org/10.1117/1.JBO.18.2.027001

[3] Y.N. Kharzheev, Phys. Part. Nucl., 50 (1), 42 (2019). https://doi.org/10.1134/S1063779619010027

[4] B. Lee, S.H. Shin, W.J. Yoo, K.W. Jang, Opt. Rev., 23 (5), 806 (2016). https://doi.org/10.1007/s10043-016-0240-8

[5] F. Therriault-Proulx, L. Beaulieu, L. Archambault, S. Beddar, Phys. Med. Biol., 58 (7), 2073 (2013). https://doi.org/10.1088/0031-9155/58/7/2073

[6] V.F. Tarasenko, E.Kh. Baksht, A.G. Burachenko, D.V. Beloplotov, A.V. Kozyrev, IEEE Trans. Plasma Sci., 45 (1), 76 (2017). https://dio.org/10.1109/TPS.2016.2637570

[7] В.И. Олешко, Е.Х. Бакшт, А.Г. Бураченко, В.Ф. Тарасенко, ЖТФ, 87 (2), 271 (2017). DOI: $10.21883 /$ JTF.2017.02.44137.1860

[8] L.D. Landau, J.S. Bell, M.J. Kearsley, L.P. Pitaevskii, E.M. Lifshitz, J.B. Sykes, Electrodynamics of continuous media (Pergamon Press, Oxford, 1984). https://www.elsevier.com/books/electrodynamics-of-continuousmedia/landau/978-0-08-030275-1

[9] V.F. Tarasenko, V.I. Oleshko, M.V. Erofeev, E.I. Lipatov, D.V. Beloplotov, M.I. Lomaev, A.G. Burachenko, E.Kh. Baksht, J. Appl. Phys., 125 (24), 244501 (2019). https://doi.org/10.1063/1.5094956

[10] V.F. Tarasenko, E.Kh. Baksht, D.V. Beloplotov, A.G. Burachenko, M.I. Lomaev, Jpn. J. Appl. Phys., 59 (SH), SHHD01 (2020). https://doi.org/10.35848/1347-4065/ab7475

[11] Е.Х. Бакшт, А.В. Вуколов, М.В. Ерофеев, Г.А. Науменко, А.П. Потылицын, В.Ф. Тарасенко, А.Г. Бураченко, М.В. Шевелев, Письма в ЖЭТФ, 109 (9), 584 (2019). DOI: $10.1134 / \mathrm{S} 0370274 \mathrm{X} 19090029$

[12] G.R. Araujo, T. Pollmann, A. Ulrich, Eur. Phys. J. C, 79 (8), 653 (2019). https://doi.org/10.1140/epjc/s10052-019-7152-2

[13] J.M. Corning, G.R. Araujo, P.C.F. Di Stefano, V. Pereymak, T. Pollmann, P. Skensved, J. Instrum., 15 (3), C03046 (2020). https://doi.org/10.1088/1748-0221/15/03/C03046 